\title{
Laser ablation of a turbid medium: modelling and experimental results
}

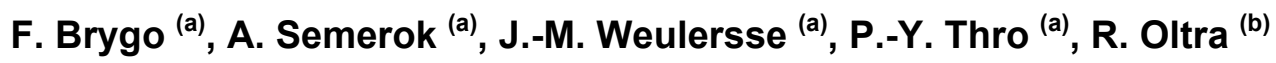

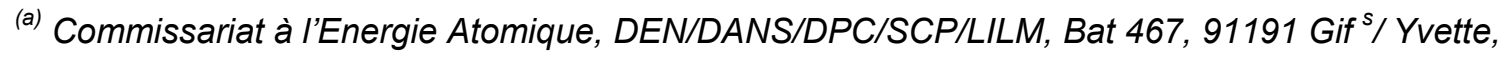 \\ France \\ (b) LRRS-UMR 5613 CNRS, Université de Bourgogne, Dijon, France
}

\begin{abstract}
Q-Switched Nd:YAG laser ablation of a turbid medium (paint) is studied. The optical properties (absorption coefficient, scattering coefficient and its anisotropy) of a paint are determined with a multiple scattering model (3-flux model), and from measurements of reflection - transmission of light through thin layers. The energy deposition profiles are calculated at wavelengths $532 \mathrm{~nm}$ and $1.064 \mu \mathrm{m}$. They are different from those described by a Lambert - Beer law. In particular, the energy deposition of the laser beam is not maximum on the surface but at some depth inside the medium. The ablated rate was measured for the two wavelengths, and compared with the energy deposition profile predicted by the model. This allows to understand the evolution of the ablated depth with the wavelength: the more the scattering coefficient is higher, the more the ablated depth and the threshold fluence of ablation decrease.
\end{abstract}




\section{Introduction}

Several studies were devoted on the laser ablation of paint for decontamination processes in the nuclear industry [1 - 7]. Nuclear dismantling faces the problem of paint removal on large surfaces of painted walls. The conventional methods for paint stripping on concrete walls are mainly based on mechanical grinder and lead to an important volume of aerosols and wastes. Laser ablation has been evaluated as a promising method for paint removal with a number of advantages: the method reduces considerably the waste volume as the removal of paint is selective, the ablated matter can be collected by aerosol aspiration/filtration, and the automation of the process can provide a higher personal safety.

Laser ablation is a complex phenomenon, involving the fast heating of the material and its ejection. The effects of the laser beam parameters on the ablation rate, as the fluence, the wavelength, the pulse duration and the repetition rate, have been studied and described in the literature [4 - 8]. However, paints are complex materials, including polymer properties and oxides (pigments) properties, and the ablation process is not completely understood. This paper focuses on the first step of the laser-matter interaction, which is the energy deposition of the laser beam in the material. From the energy deposition in depth profile, the laser ablation properties, i.e. the threshold fluence, the ablated rate and the ablation efficiency, will be analysed.

Paint is a turbid medium. The multiple scattering of the light in the bulk leads to a particular energy deposition of the laser beam in the medium, which differs from the Lambert - Beer law. In particular, overheating inside the bulk were predicted [9] and measured [10]. The laser ablation efficiency and optical properties of a turbid medium were already compared [11] for sintered polytetrafluoroethylene. Other works are reported for the ablation of tissue [9, 12]. However, for paint, no study relates to the characterization of the energy deposition, compared to the ablation rate, despite that optical properties are characterized for studies in conservation [13], on solar panels [14 17], on the ageing of paint in space [18], or on the scattering of light by pigments [19, 20].

$\mathrm{Nd}$ :YAG laser ablation of a grey epoxy paint, at wavelengths $532 \mathrm{~nm}$ and $1.064 \mu \mathrm{m}$, are presented in this paper. The absorption coefficient, scattering coefficient and its anisotropy are determined with a 3-flux model and from optical measurements of transmitted and diffuse flux. The measured parameters and the model allow calculating the energy deposition profile. The ablation rates are measured for the two wavelengths, and compared with these specific energy deposition profiles. 


\section{3-flux model}

The 3-flux model $[21,22]$ is applied to describe the light propagation in a turbid medium and to determine the energy deposition profile. The fundamental principles of the model are presented below:

One considers the flux $F_{1}$, representing the laser (collimated) beam travelling in the positive direction $+\mathrm{z}$ in a turbid medium. Within a differential distance $\mathrm{dz}$, the collimated flux can be transferred in diffuse flux in the same direction $\left(\mathrm{F}_{2}\right)$ and in the opposite direction $\left(\mathrm{F}_{3}\right)$, and the diffuse flux $\mathrm{F}_{2}$ and $\mathrm{F}_{3}$ can be exchanged (cf. Figure 1). Five parameters are introduced: $k$, the absorption coefficient of the collimated flux; $\mathrm{K}$, the absorption coefficient of the diffuse flux; $\mathrm{S}_{1}$, the scattering coefficient from the collimated flux to the diffuse flux in the direction $+\mathrm{z} ; \mathrm{S}_{2}$, the scattering coefficient from the collimated flux to the diffuse flux in the direction $-\mathrm{z}$; and $\mathrm{S}$, the scattering coefficient from a diffuse flux to the other.

The 3-flux equations are:

$$
\begin{aligned}
& \frac{\mathrm{dF}_{1}}{\mathrm{dz}}=-\left(\mathrm{k}+\mathrm{S}_{1}+\mathrm{S}_{2}\right) \mathrm{F}_{1} \\
& \frac{\mathrm{dF}_{2}}{\mathrm{dz}}=\mathrm{S}_{1} \mathrm{~F}_{1}-(\mathrm{K}+\mathrm{S}) \mathrm{F}_{2}+\mathrm{SF}_{3} \\
& -\frac{\mathrm{dF}_{3}}{\mathrm{dz}}=\mathrm{S}_{2} \mathrm{~F}_{1}+\mathrm{SF}_{2}-(\mathrm{K}+\mathrm{S}) \mathrm{F}_{3}
\end{aligned}
$$

These equations are one-dimensional, which imposes that the laser beam diameter is much larger than the thickness e of the medium, $1 / \mathrm{k}$ or $1 / \mathrm{S}$, which represent characteristic absorption length and diffusion length respectively. Solutions to this set of equations are:

$$
\begin{aligned}
& \mathrm{F}_{1}=\mathrm{C}_{1} \mathrm{e}^{-\lambda z} \\
& \mathrm{~F}_{2}=\mathrm{C}_{1} \mathrm{~A}_{1} \mathrm{e}^{-\lambda z}+\mathrm{C}_{2}(1+\beta) \mathrm{e}^{-\sigma z}+\mathrm{C}_{3}(1-\beta) \mathrm{e}^{\sigma z} \\
& \mathrm{~F}_{3}=\mathrm{C}_{1} \mathrm{~A}_{2} \mathrm{e}^{-\lambda z}+\mathrm{C}_{2}(1-\beta) \mathrm{e}^{-\sigma z}+\mathrm{C}_{3}(1+\beta) \mathrm{e}^{\sigma z}
\end{aligned}
$$

with

$$
\begin{gathered}
\lambda=\mathrm{k}+\mathrm{S}_{1}+\mathrm{S}_{2} ; \pm \sigma= \pm \sqrt{\mathrm{K}(\mathrm{K}+2 \mathrm{~S})} \\
\mathrm{A}_{1}=\frac{\mathrm{SS}_{2}+(\mathrm{K}+\mathrm{S}+\lambda) \mathrm{S}_{1}}{\sigma^{2}-\lambda^{2}} ; \mathrm{A}_{2}=\frac{\mathrm{SS}_{1}+(\mathrm{K}+\mathrm{S}-\lambda) \mathrm{S}_{2}}{\sigma^{2}-\lambda^{2}} ; \beta=\sqrt{\frac{\mathrm{K}}{\mathrm{K}+2 \mathrm{~S}}}
\end{gathered}
$$


Coefficients $\mathrm{C}_{1}, \mathrm{C}_{2}$ and $\mathrm{C}_{3}$ are determined from the boundary conditions at $\mathrm{z}=\mathrm{e}$ and $\mathrm{z}=0 . \mathrm{C}_{1}$ represents the laser flux penetrating into the medium. One imposes $C_{1}=1 . C_{2}$ and $C_{3}$ are determined from the reflections at the interfaces. They are calculated as being:

$$
\begin{aligned}
& C_{2}=\frac{-\left(1+\beta+(\beta-1) R_{e}\right)\left(A_{1}-R_{0} A_{2}\right)+e^{-\lambda e-\sigma e}\left(R_{e} A_{1}-A_{2}\right)\left(\beta-1+R_{0}+R_{0} \beta\right)}{\left(1+\beta+(\beta-1) R_{e}\right)\left(1+\beta+(\beta-1) R_{0}\right)-e^{-2 \sigma e}\left(\beta-1+R_{e}+\beta R_{e}\right)\left(\beta-1+R_{0}+\beta R_{0}\right)} \\
& C_{3}=\frac{\left(\beta-1+R_{e}+\beta R_{e}\right)\left(R_{0} A_{2}-A_{1}\right)-e^{\sigma e-\lambda e}\left(A_{2}-A_{1} R_{e}\right)\left(1+\beta+(\beta-1) R_{0}\right)}{e^{2 \sigma e}\left(1+\beta+(\beta-1) R_{e}\right)\left(1+\beta+(\beta-1) R_{0}\right)-\left(\beta-1+R_{e}+\beta R_{e}\right)\left(\beta-1+R_{0}+\beta R_{0}\right)}
\end{aligned}
$$

The reflection coefficients for the diffuse flux at the interfaces $z=0\left(R_{0}\right)$ and $z=e\left(R_{e}\right)$ correspond to an average value of the reflection for angle of incidence between 0 and $\pi / 2$. The reflection of the collimated beam at the interface $z=e$ is supposed negligible $(<5 \%$ in our case).

The measured reflection and transmission of the light through thin layers are distinguishable in 3 coefficients: the diffuse reflection $\mathrm{R}_{\text {diffuse }}$, the diffuse transmission $\mathrm{T}_{\text {diffuse }}$ and the transmission of the collimated beam $\mathrm{T}_{\text {collimated. }}$ The diffuse reflection represents only the bulk reflection. The surface reflections (e.g. the specular reflection) are not considered (one supposed with $\mathrm{C}_{1}=1$ that the total laser beam energy penetrates in the medium). We have:

$$
\begin{aligned}
& \mathrm{R}_{\text {diffuse }}=\mathrm{F}_{3}(0)-\mathrm{F}_{2}(0) \\
& \mathrm{T}_{\text {diffuse }}=\mathrm{F}_{2}(\mathrm{e})-\mathrm{F}_{3}(\mathrm{e}) \\
& \mathrm{T}_{\text {collimated }}=\mathrm{F}_{1}(\mathrm{e})
\end{aligned}
$$

The unknown parameters $\mathrm{k}, \mathrm{K}, \mathrm{S}, \mathrm{S}_{1}, \mathrm{~S}_{2}$ can be resumed to 3 unknown parameters. The reference [21] showed that the absorption of the diffuse flux is close to twice the absorption of the collimated flux: $\mathrm{K} \approx 2 \mathrm{k}$. This difference is related to a larger optical path of the diffuse flux compared to the collimated flux, according to the axis $\mathrm{z}$. The coefficients $\mathrm{S}_{1}$ and $\mathrm{S}_{2}$ are in relation with the phase function $\mathrm{p}(\cos \theta)$ [22], which describes the angular properties of the scattering. The phase function is normalised as:

$$
\frac{1}{2} \int_{-1}^{1} \mathrm{p}(\cos \theta) \mathrm{d}(\cos \theta)=1
$$

with $\theta$ the angle between the direction of the incident beam and the scattered beam. By introducing the global scattering coefficient s, we have:

$$
\mathrm{S}=\mathrm{S}_{1}+\mathrm{S}_{2}
$$


$\mathrm{S}_{1}$ expresses the forward diffusion, i.e. the whole light scattered by a particle and propagating in the positive half space $+\mathrm{z}$ :

$$
\mathrm{S}_{1}=\frac{\mathrm{s}}{2} \int_{0}^{1} \mathrm{p}(\cos \theta) \mathrm{d}(\cos \theta)
$$

And, $S_{2}$, for the negative half space:

$$
\mathrm{S}_{2}=\frac{\mathrm{s}}{2} \int_{-1}^{0} \mathrm{p}(\cos \theta) \mathrm{d}(\cos \theta)
$$

If we express the phase function as a series of Legendre polynomials $P_{n}(\cos \theta)$, as

$$
\mathrm{p}(\cos \theta)=\sum_{\mathrm{n}=0}^{\infty} \mathrm{g}_{\mathrm{n}} \mathrm{P}_{\mathrm{n}}(\cos \theta)
$$

one gets:

$$
\mathrm{S}_{1}=\frac{\mathrm{s}}{2}\left(1+\frac{\mathrm{g}_{1}}{2}-\frac{\mathrm{g}_{3}}{8}+\ldots\right)
$$

with $g_{i}$ calculated as:

$$
\mathrm{g}_{\mathrm{n}}=\left(\mathrm{n}+\frac{1}{2} \int_{-1}^{+1} \mathrm{p}(\cos \theta) \mathrm{P}_{\mathrm{n}}(\cos \theta) \mathrm{d}(\cos \theta)\right.
$$

Thereafter, one considers only the first order of the development in the equation (10), (i.e. parameter $\mathrm{g}_{1}$ ), because the other terms are negligible compared to it, or are divided by a more important factor as seen in (11). The reference [21] showed that coefficients $S$ and $s$ are related as:

$$
\mathrm{S}=\mathrm{s}\left(3 \mathrm{~g}_{0}-\mathrm{g}_{1}\right) / 4
$$

Since the values of the three unknown parameters $\mathrm{k}, \mathrm{s}$, and $\mathrm{g}_{1}$, representing the absorbing coefficient, the scattering coefficient and its anisotropy, are determined, the energy deposition profile is calculated as:

$$
\mathrm{M}(\mathrm{z})=\mathrm{kF}_{1}+\mathrm{KF}_{2}+\mathrm{KF}_{3}
$$

The energy deposition profile is related to the heat source term $Q(x, y, z, t)$ usually introduced in the heat equation to calculate the temperature evolution of the material [8], as $Q(x, y, z, t)=(1-R)$ $M(z) I_{0}(x, y, t)$, with $R$ the reflection at the surface. For non-scattering semi-infinite matter, the energy deposition profile is normalised as:

$$
\int_{0}^{\infty} \mathrm{M}(\mathrm{z}) \mathrm{dz}=1
$$


Relation (15) can't be used with the relation (14), because one part of the laser beam is reemitted from the surface as a diffuse reflection (coming from the backward flux $F_{3}$ ). For the relation (14), with a semi-infinite medium, one have:

$$
\int_{0}^{\infty} \mathrm{M}(\mathrm{z}) \mathrm{dz}=1-\mathrm{R}_{\text {diffuse }}
$$

The parameters $\mathrm{k}, \mathrm{s}$ and $\mathrm{g}_{1}$ must be known to calculate the energy deposition profile. They can be determined from measurements of the diffuse reflection, diffuse transmission and collimated transmission of light through thin layers.

\section{Measurement of the optical properties of the paint}

Homogeneous thin layers of paint of a few tens of micrometers were realised to measure the diffuse reflection, diffuse transmission and collimated transmission of a laser beam. The thin layers were fabricated by "spin coating", on a transparent polycarbonate substrate (lexan). A characteristic profile of the thin layers is given on figure 2 .

The diffuse reflection, collimated transmission and diffuse transmission are measured with an integrating sphere "Labsphere" and a sensor "Centronic TO5". The internal reflection coefficient of the integrating sphere was calibrated before the measurements. Two lasers are used for measurements: a He:Ne laser at $\lambda=543.5 \mathrm{~nm}$, and a Nd:YAG laser at $\lambda=1.064 \mu \mathrm{m}$. Results are supposed to be the same at the wavelength $\lambda=532 \mathrm{~nm}$ and the wavelength $\lambda=543.5 \mathrm{~nm}$. The 3-flux model parameters $\mathrm{k}$, $\mathrm{s}$ and $\mathrm{g}_{1}$ are fitted from the experimental results. At $\lambda=543.5 \mathrm{~nm}$, the measurements and the theoretical transmissions and reflections as a function of the thickness of the paint are presented on figure 3, with the best fitted parameters $\mathrm{s}=1.8 \times 10^{5} \mathrm{~m}^{-1}, \mathrm{k}=1.2 \times 10^{4} \mathrm{~m}^{-1}, \mathrm{~g}_{1}=1.45$. In the same way, the transmissions and reflection as a function of the thickness of the paint are presented on figure 4 at $\lambda=1.064 \mu \mathrm{m}$, with the best fitted parameters $\mathrm{s}=9 \times 10^{4} \mathrm{~m}^{-1}, \mathrm{k}=9 \times 10^{3} \mathrm{~m}^{-1}, \mathrm{~g}_{1}=1.8$.

The scattering coefficient is twice more important at $\lambda=543.5 \mathrm{~nm}$ than at $\lambda=1.064 \mu \mathrm{m}$. This is due to the diameter of the scattering pigments $\left(\mathrm{TiO}_{2}\right)$. One can estimate the scattering coefficient of the medium by taking into account the mean diameter of the particles, measured to be close to $350 \mathrm{~nm}$, and with the volume proportion $\sim 0.8 \%$. The ordinary index of refraction of $\mathrm{TiO}_{2}\left(\mathrm{n}_{0}\right)$ is 2.66 , and the extraordinary index of refraction $\left(\mathrm{n}_{\mathrm{e}}\right)$ is 2.96 at $543 \mathrm{~nm}$ [23]. By assuming that $\mathrm{TiO}_{2}$ is isotropic, its index of refraction is calculated as $n_{\mathrm{TiO} 2}=\left(2 n_{0}+n_{e}\right) / 3=2.76$ at $543 \mathrm{~nm}$. In the same way, the 
index of refraction of $\mathrm{TiO}_{2}$ is 2.57 at $1.064 \mu \mathrm{m}$. From these values, the scattering coefficient of the medium is calculated as the scattering coefficient of a single particle multiplied by its volume proportion in the material. The scattering coefficient of a single particle is calculated from the Mie theory [22], with the refraction index of the polymer measured as being close to 1.5 for both wavelengths. That gives $\mathrm{s} \approx 1.6 \times 10^{5} \mathrm{~m}^{-1}$ for $\lambda=543.5 \mathrm{~nm}$ and $\mathrm{s} \approx 7 \times 10^{4} \mathrm{~m}^{-1}$ for $\lambda=1.064 \mu \mathrm{m}$. These values should be understood as estimations, because of the complexity of the medium, but they are closed to the ones obtained from the measurements and the 3-flux model.

The absorption coefficient is nearly the same for the two wavelengths. It's due to the absorbing pigments (carbon particles) that are completely opaque to the laser radiation. Thus, no difference is expected between the two wavelengths, which is well verified from the measurements and the 3-flux theory.

Finally, figure 5 represents the energy deposition profile $M(z)$ in a semi-infinite medium calculated from the 3-flux model and the previous optical parameters for the two wavelengths.

\section{Laser ablation}

The ablation of the paint was performed with Q-switched Nd:YAG lasers. The pulse duration is $80 \pm 10 \mathrm{~ns}(\mathrm{FWHM})$, the repetition rate $20 \mathrm{~Hz}$, and the wavelengths $\lambda=1.064 \mu \mathrm{m}$ and $\lambda=532 \mathrm{~nm}$. The spatial distribution of the beam intensity was homogenised by a multimode optical fibre. Details of the systems are presented in the reference [5]. The crater depths and spatial profiles are measured with a profilometer (MAHR) with a mechanical sensor (MFW-250). The depth resolution of the measurements is better than $1 \mu \mathrm{m}$. The crater spatial profiles are directly correlated with the beam profile (flat top, with the same diameter that the laser beam), as seen on figure 6 . Thus, the presented crater depths are an average value of the depth measured in different zones of the craters.

The craters depth evolution with the fluence is presented on figure 7 . The depth is proportional to the number of applied pulses in the whole studied range ( $3-100$ pulses). The threshold fluence is $1.7 \mathrm{~J} \mathrm{~cm}^{-2}$ at $\lambda=1.064 \mu \mathrm{m}$ and $1.2 \mathrm{~J} \mathrm{~cm}^{-2}$ at $\lambda=532 \mathrm{~nm}$. Over this fluence, the ablated depth increases until reaching a saturation. The maximum available depth is higher at $\lambda=1.064 \mu \mathrm{m}(13 \mu \mathrm{m} / \mathrm{pulse})$ than at $\lambda=532 \mathrm{~nm}(6.5 \mu \mathrm{m} /$ pulse $)$. 


\section{Discussion}

The thermal conductivity of the paint was estimated as being $D=0.03 \mathrm{~cm}^{2} \mathrm{~s}^{-1}[7]$. The thermal diffusion length during the laser pulse $\sqrt{\mathrm{Dt}} \approx 0.5 \mu \mathrm{m}$ is low compare to the optical penetration depth $(\sim 10 \mu \mathrm{m})$, which involves that the thermal diffusion during the pulse can be neglected. Thus, the ablation depth should be related to the profile of the energy deposition $\mathrm{M}(\mathrm{z})$ in the material. The ablation threshold fluence should depend on the laser energy deposited on the surface, and the ablated depth should depend on the optical penetration depth. Figure 5 shows that the maximum of the energy deposition is not located at the surface but inside the medium. By increasing the scattering coefficient from $\mathrm{s}=0.9 \times 10^{5} \mathrm{~m}^{-1}(\lambda=1.064 \mu \mathrm{m})$ to $\mathrm{s}=1.8 \times 10^{5} \mathrm{~m}^{-1}(\lambda=532 \mathrm{~nm})$, the total quantity of

energy absorbed in the medium $\int_{0}^{\infty} \mathrm{M}(\mathrm{z}) \mathrm{dz}$ decreases, the penetration depth decreases, and the maximum of the energy deposition increases. This can be also seen on Figure 8 where the energy deposition in a medium with a constant absorption coefficient $\mathrm{k}=1 \times 10^{4} \mathrm{~m}^{-1}$ is calculated for different scattering coefficient ranging from $\mathrm{s}=1 \times 10^{4} \mathrm{~m}^{-1}$ to $\mathrm{s}=1 \times 10^{6} \mathrm{~m}^{-1}\left(\mathrm{~g}_{1}=0\right)$.

Table 1 compares the energy deposition in the paint with the maximum available ablation depth and with the threshold fluences, for the two wavelengths. The energy deposition profile is characterized by the penetration depth, defined as $\mathrm{z}_{\mathrm{M}(0) / \mathrm{e}}$, and by the position of the maximum of the energy deposition $\mathrm{z}_{\mathrm{Mmax}}$ (cf. notation on figure 5). $\mathrm{z}_{\mathrm{M}(0) / \mathrm{e}}$ is the depth at which the energy deposition is equal to $1 / \mathrm{e}$ of the energy deposition at the surface. The value of the energy deposition is represented by the terms $\mathrm{M}(0)$ and $\mathrm{M}_{\max }$. One notices that a proportional relation is found between these values and the ablation measurements: by decreasing the penetration depth of a factor $\sim 1.9$ between $1.064 \mu \mathrm{m}$ and $532 \mathrm{~nm}$, the ablation depth decreases by a factor 2 . Moreover, by decreasing the value of the energy deposition at the surface (or close to the surface) by a factor $\sim 0.6$, the threshold fluence decreases of a factor $\sim 0.7$. The same proportionality is obtained by considering the position of the maximum of the energy deposition $z_{M \max }$ and the value $M_{\max }$ instead of $z_{M(0) / e}$ and $M(0)$. The maximum available ablation depths are close to the depths of the maximum of the energy depositions $\mathrm{Z}_{\mathrm{Mmax}}$ for both wavelengths.

Thus, one highlights that the maximum ablation depth is more important with a low scattering material, as well as the global absorbed energy. However, the maximum ablation efficiencies (i.e. the 
ablated volume per joule) are nearly the same for the two wavelengths. The ablation efficiencies are presented on figure 9. Because the craters have flat top profiles with the same diameter as the laser beam, the ablation efficiency $\left(\mathrm{mm}^{3} \mathrm{~J}^{-1}\right)$ is the depth per pulse, presented on figure 7 , divided by the fluence. The maximum efficiency is close to $\eta=0.25 \mathrm{~mm}^{3} \mathrm{~J}^{-1}$, for the fluence $F=2.2 \mathrm{~J} \mathrm{~cm}^{-2}$ at $532 \mathrm{~nm}$ and $\eta=0.22 \mathrm{~mm}^{3} \mathrm{~J}^{-1}$ for the fluence $F=3.5-4.5 \mathrm{~J} \mathrm{~cm}^{-2}$ at $1.064 \mu \mathrm{m}$. These similar efficiencies can be well understood by the modelling of the laser beam energy deposition in the bulk: the increase of the scattering of the paint from the wavelength $1.064 \mu \mathrm{m}$ to $532 \mathrm{~nm}$ decreases the ablation threshold at the same time that the ablated depth decreases. Of course, for industrial application of a decontamination process, the wavelength $\lambda=1.064 \mu \mathrm{m}$ should be highlight, because the available power is higher than at $\lambda=532 \mathrm{~nm}$, for similar cost.

\section{Conclusion}

$\mathrm{Nd}$ :YAG laser ablation of a turbid medium (paint) was studied at the wavelengths $1.064 \mu \mathrm{m}$ and $532 \mathrm{~nm}$. The paint scattering and absorption coefficients were determined with a 3-flux model and from measurements of optical transmission and reflection of light through thin layers. The energy deposition profiles were calculated with the obtained optical parameters and compared with the ablation depth and ablation threshold. Variation of the threshold fluence and maximum available ablation depth per pulse with the wavelength are well explained by the energy deposition profile. More particularly, one observes that a higher scattering coefficient decreases the total absorbed energy, decreases the penetration depth, and increases the maximum of the energy deposition. This leads to lower threshold fluence and lower ablated depth. Thus, for this paint, the ablation efficiencies are similar for the two wavelengths. Additional experiments and studies will be performed on the link between the absolute value of the ablation rate and the energy deposition profile. In particular, the influence of the overheating inside the bulk due to the multiple scattering will be studied in future work.

\section{Acknowledgment}

This research is financially supported by AREVA-COGEMA. The authors would like to express their gratitude to Mr G. Decobert and Mr H. Masson for fruitful discussions. 


\section{References}

[1] M.J.J. Schmidt, L. Li, J.T. Spencer, P.H. Key, Appl. Surf. Science, 138 - 139, 418 (1999).

[2] D.E. Roberts. Conference Digest. Conference on Lasers and Electro Optics Europe IEEE, Piscataway, NJ, USA (2000).

[3] M.J.J. Schmidt, L. Li, J.T. Spencer, J. Mat. Proc. Tech, 138, 109 (2003).

[4] F. Brygo, C. Dutouquet, C. Lascoutouna, F. Le Guern, A. Semerok, J.M. Weulersse, R. Oltra, $18^{\text {th }}$ International conference on Surface Modification Technologies. Edited by T.S. Sudarshan and M. Jeandin. Dijon, (2004).

[5] F. Brygo, Ch. Dutouquet, F. Le Guern, R. Oltra, A. Semerok, J.M. Weulersse, Applied Surface Science, 252/6, 2131 (2006).

[6] F. Brygo, A. Semerok, J.M. Weulersse, P.Y. Thro, R. Oltra, G. Decobert, Proceedings of the European Nuclear Conference 2005, Session 11 (Dismantling), Versailles (2005).

[7] F. Brygo, A. Semerok, R. Oltra, J.-M. Weulersse, S. Fomichev, Applied Surface Science (2006). In press.

[8] Laser Processing and Chemistry, D. Bauerle. Edition Springer, Berlin. $3^{\text {rd }}$ edition (2000).

[9] S.L. Jacques, S.A. Prahl. Lasers in surgery and medicine 6, 494 (1987).

[10] A. A. Karabutov, I.M. Pelivanov, N. B. Podymova, S. E. Skipetrov, JETP Letters, 70/3, 183 (1999).

[11] N. Huber, J. Heitz, D. Bäuerle, Eur. Phys. J. Appl. Phys, 25, 33 (2004).

[12] Laser-Tissue interactions. Fundamentals and Applications. M. Niemz. Ed. Springer, Berlin (1996).

[13] S.R. Jones, Studies in conservation 38, 174 (1993).

[14] M.K. Gunde, Z.C. Orel, Appl. Optics, 39, 4 (2000).

[15] M.K. Gunde, Z.C. Orel, M.G. Hutchins, Solar Energy materials and solar cells 80, 239 (2003).

[16] T. Tesfamichael, A. Hoel, E. Wäckelgård, G.A. Niklasson, M.K. Gunde, Z.C. Orel, Solar Energy 69 (sup) 35 (2000).

[17] Z.C. Orel, M.K. Gunde, B. Orel, M. Köhl, EuroSun'96, 500 (1996).

[18] C. Tonon, C. Rozé, T. Girasole, M. Dinguirard, Applied Optics, 40/22, 3718 (2001).

[19] L.E. McNeil, R.H. French, J. Appl. Phys, 89/1, 283 (2001).

[20] L.E. McNeil, R.H. French, Acta Mater, 48, 4571 (2000).

[21] P.S. Mudgett, L.W. Richards, Appl. Optics, 10/7, 1485 (1971).

[22] Wave propagation and scattering in random media. A. Ishimaru. Academic Press (1978). 
[23] CRC materials science and engineering handbook. J.F. Shackelford, W. Alexander, $3^{\text {rd }}$ ed CRC Press (2001). 


\section{Table}

Table 1: Comparison between the energy deposition, the ablation depth and the threshold fluence for the two wavelengths.

\begin{tabular}{c|ccc|ccc}
\hline & $\mathbf{M}(\mathbf{0})\left(\mathbf{m}^{-1}\right)$ & $\mathbf{M}_{\text {max }}\left(\mathbf{m}^{-1}\right)$ & $\begin{array}{c}\text { Threshold } \\
\text { fluence }\left(\mathbf{J ~ c m}^{-2}\right)\end{array}$ & $\mathbf{z}_{\text {Mmax }}(\mathbf{m})$ & $\begin{array}{c}\text { Absorption depth } \\
\mathbf{M}(\mathbf{0}) / \mathbf{e}(\mathbf{m})\end{array}$ & $\begin{array}{c}\text { Maximum ablated } \\
\text { depth }(\boldsymbol{\mu m})\end{array}$ \\
\hline $\mathbf{1 . 0 6 4} \mathbf{\mu m}$ & $1.43 \times 10^{4}$ & $1.72 \times 10^{4}$ & 1.7 & $8 \times 10^{-6}$ & $5.2 \times 10^{-5}$ & 13 \\
$\mathbf{5 3 2} \mathbf{~ n m}$ & $2.28 \times 10^{4}$ & $2.64 \times 10^{4}$ & 1.2 & $4.1 \times 10^{-6}$ & $2.84 \times 10^{-5}$ & 6.5 \\
\hline $\mathbf{1 . 0 6 4 / 5 3 2}$ & 0.63 & 0.65 & $1 / 0.71$ & 1.95 & 1.83 & 2 \\
\hline
\end{tabular}




\section{Figures captions}

Figure 1: Scheme of the 3-flux model.

Figure 2: Characteristic profile of a thin layer.

Figure 3: Collimated transmission, diffuse transmission and diffuse reflection for various thicknesses at $\lambda=532 \mathrm{~nm}$. Lines: 3-flux model with $\mathrm{s}=1.8 \times 10^{5} \mathrm{~m}^{-1}, \mathrm{k}=1.2 \times 10^{4} \mathrm{~m}^{-1}, \mathrm{~g}_{1}=1.45$. Symbols : Experimental results.

Figure 4: Collimated transmission, diffuse transmission and diffuse reflection for various thicknesses at $\lambda=1.064 \mu \mathrm{m}$. Lines: 3-flux model with $\mathrm{s}=9 \times 10^{4} \mathrm{~m}^{-1}, \mathrm{k}=9 \times 10^{3} \mathrm{~m}^{-1}, \mathrm{~g}_{1}=1.8$. Symbols : Experimental results.

Figure 5: Energy deposition profile in the paint at $\lambda=1.064 \mu \mathrm{m}\left(\mathrm{s}=9 \times 10^{4} \mathrm{~m}^{-1}, \mathrm{k}=9 \times 10^{3} \mathrm{~m}^{-1}, \mathrm{~g}_{1}=1.8\right)$ and $\lambda=532 \mathrm{~nm}\left(\mathrm{~s}=1.8 \times 10^{5} \mathrm{~m}^{-1}, \mathrm{k}=1.2 \times 10^{4} \mathrm{~m}^{-1}, \mathrm{~g}_{1}=1.45\right)$ from the 3 -flux model.

Figure 6: Typical crater profile, obtained at $F=4 \mathrm{~J} \mathrm{~cm}^{-2}, \lambda=1.064 \mu \mathrm{m}, 10$ pulses.

Figure 7: Ablated depth per pulse as a function of the fluence for $\lambda=1.064 \mu \mathrm{m}$ and $\lambda=532 \mathrm{~nm}$.

Figure 8: Energy deposition profile from the 3-flux model with $\mathrm{k}=1 \times 10^{4} \mathrm{~m}^{-1}, \mathrm{~g}_{1}=0$, and for various $\mathrm{s}$.

Figure 9: Ablation efficiencies as a function of the fluence for the two wavelengths. 
Figures

Figure 1

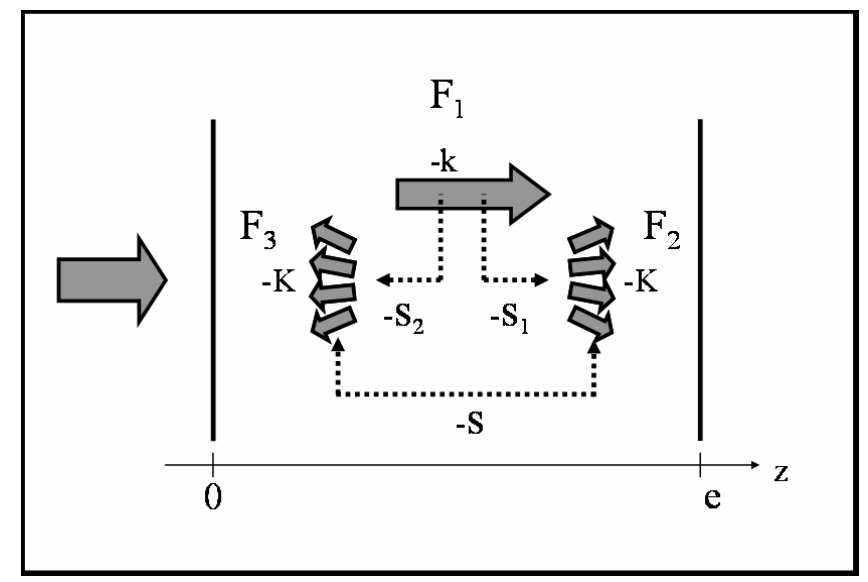


Figure 2

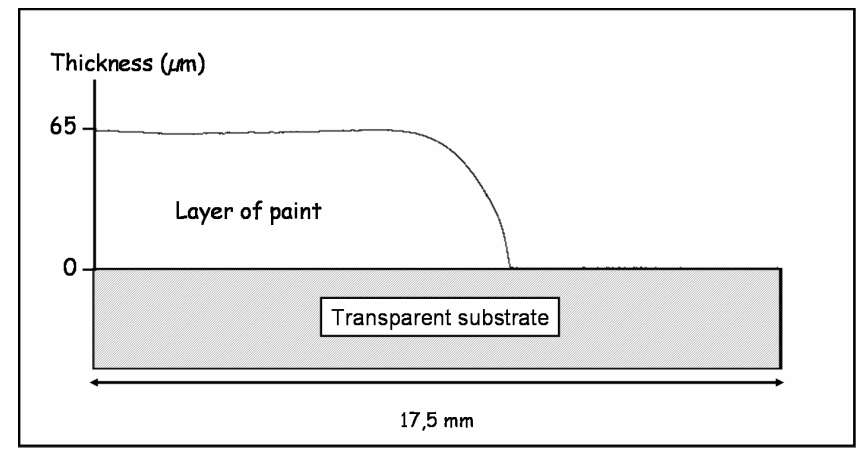


Figure 3

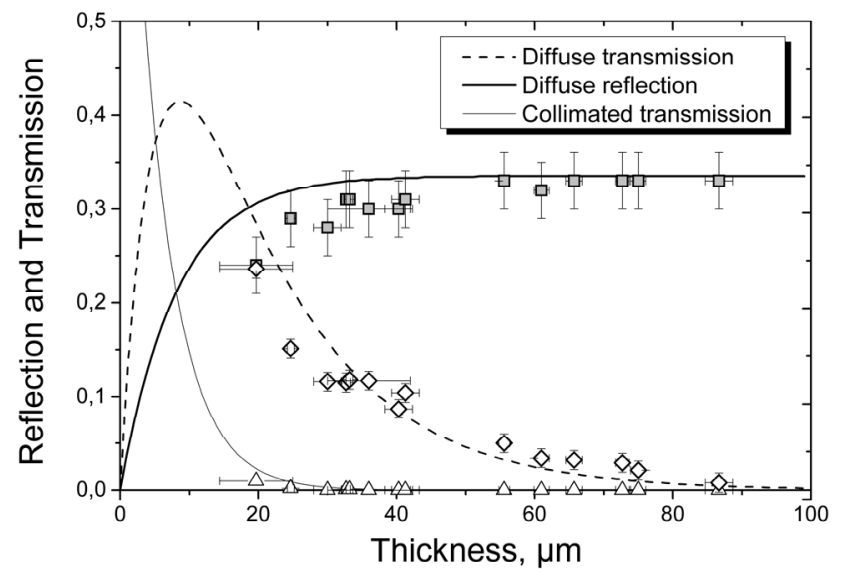


Figure 4

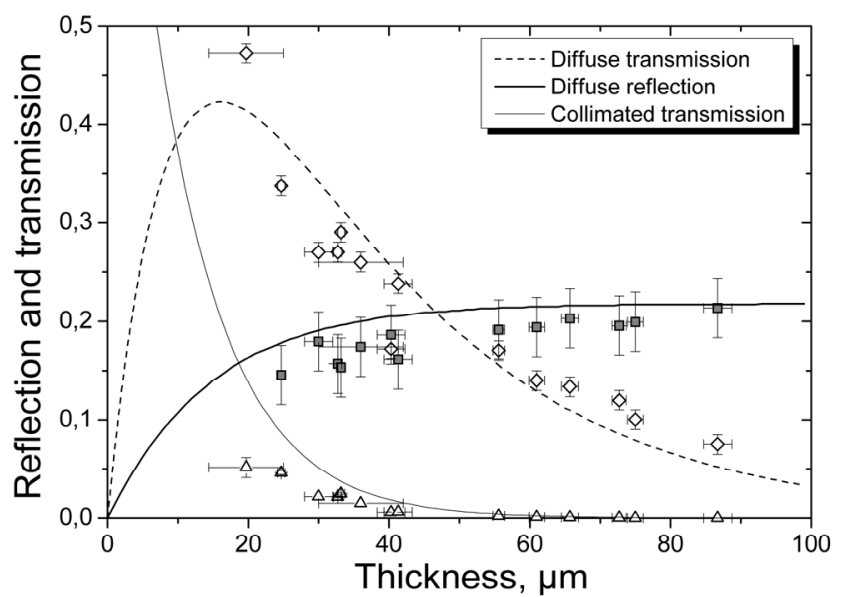


Figure 5

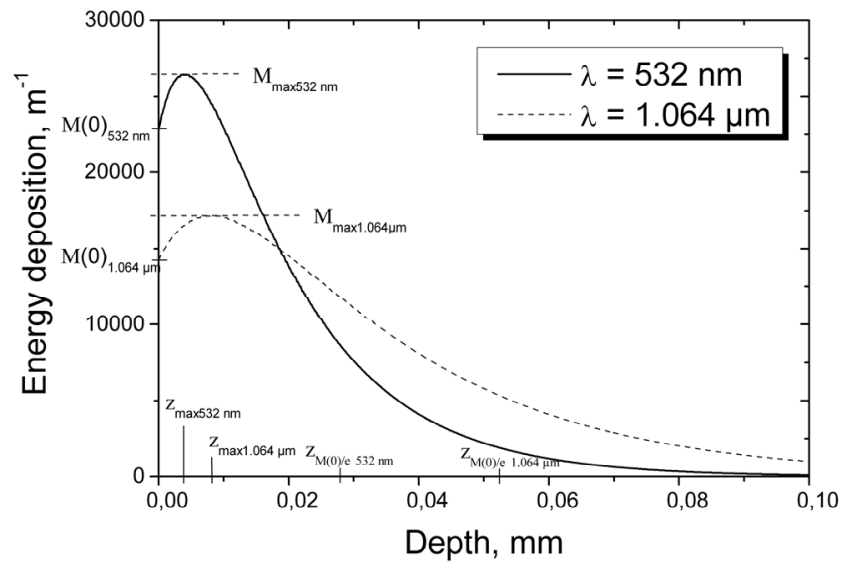


Figure 6:

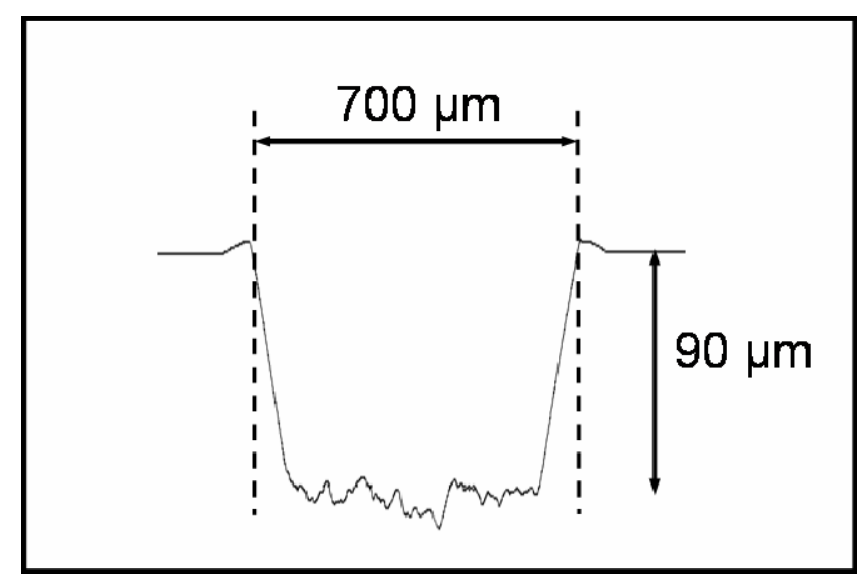


Figure 7

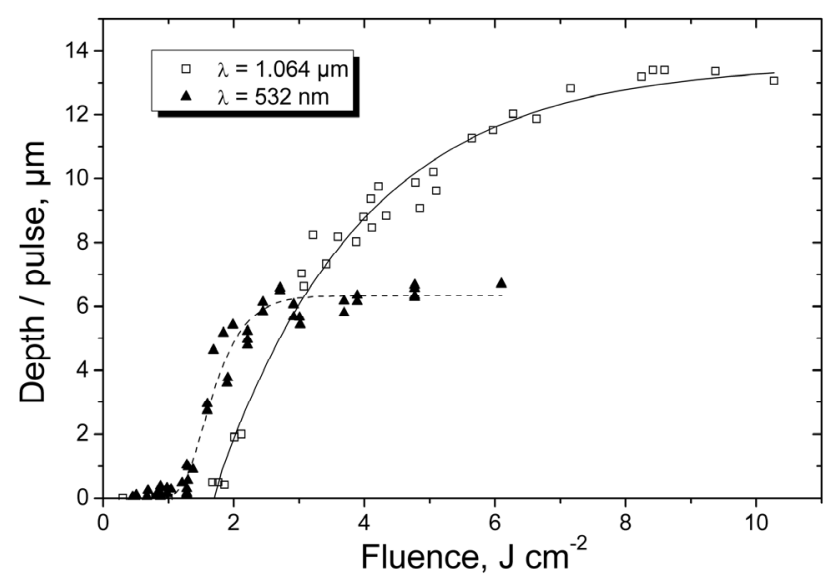


Figure 8

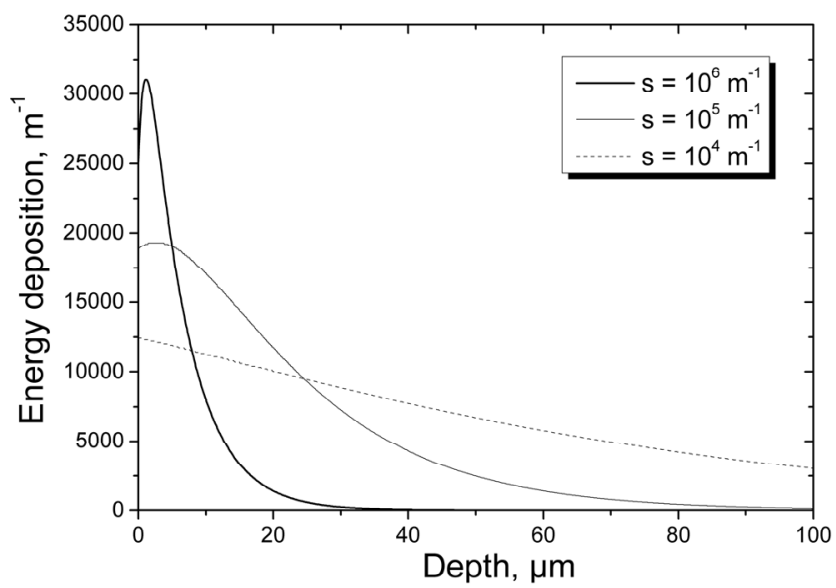


Figure 9

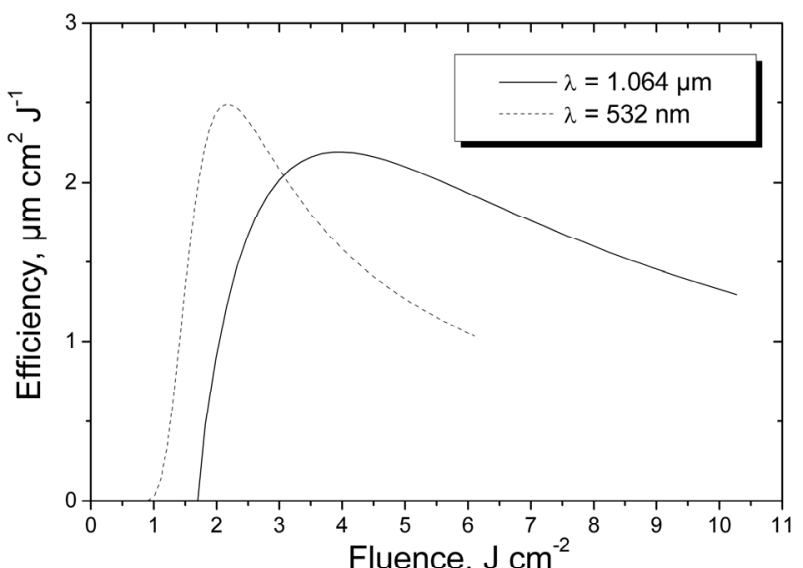

